\title{
Pediatric Pneumococcal Hemolytic Uremic Syndrome Treated with Sequence Tandem Therapeutic Plasma Exchange and Continuous Venovenous Hemodiafiltration: A Case Report
}

\author{
Josko Markic ${ }^{1,2}$ Branka Polic $^{1,2}$ Tanja Kovacevic ${ }^{1}$ Marijana Rogulj ${ }^{1}$ Tatjana Catipovic Ardalic ${ }^{1}$ \\ ${ }^{1}$ Department of Pediatrics, University Hospital of Split, Split, Croatia \\ 2 Department of Pediatrics, University of Split School of Medicine, \\ Split, Croatia \\ Address for correspondence Josko Markic, MD, PhD, Department of \\ Pediatrics, University Hospital of Split, Spinciceva 1, 21000 Split, \\ Croatia (e-mail: josko.markic@gmail.com). \\ J Child Sci 2020;10:e221-e223.
}

\begin{abstract}
Hemolytic uremic syndrome (HUS) is characterized by microangiopathic hemolytic anemia, thrombocytopenia, and acute kidney injury. Approximately $5 \%$ of HUS cases are associated with Streptococcus pneumoniae infections (pHUS). Treatment includes supportive care with appropriate antimicrobial therapy, fluid and blood product resuscitation, and renal replacement therapy. We presented a case of a 22-monthold previously healthy girl, who was hospitalized at University Hospital of Split. Leftsided pneumonia and sepsis caused by S. pneumoniae were confirmed. The course of illness was complicated with development of pHUS. Since the pathogenesis of pHUS is

\section{Keywords}

- hemolytic uremic syndrome

- child

- streptococcus pneumonia

- plasmapheresis

- therapeutic only partially understood, the treatment remains controversial. Our patient was successfully treated with daily sequence tandem continuous venovenous hemodiafiltration and therapeutic plasma exchange with albumins, along with other supportive measures. Therefore, in our opinion, plasmapheresis should be considered as a part of standard treatment of children with pHUS. Additionally, the incidence of pHUS appears to be increasing. S. pneumoniae is a particularly important among pediatric pathogens and it can cause wide spectrum of illnesses. Therefore, due to the significant burden of invasive pneumococcal disease, pneumococcal vaccination should be encouraged.
\end{abstract}

\section{Introduction}

In childhood, hemolytic uremic syndrome (HUS) is usually triggered by infection and is characterized by microangiopathic hemolytic anemia, thrombocytopenia, and acute kidney injury (AKI). The vast majority of cases are preceded by an intestinal infection caused by Shiga-like toxin producing Escherichia coli (STEC). ${ }^{1}$ Atypical HUS (aHUS) is more severe form of HUS that results from the over activation of the alternative complement pathway. ${ }^{2}$ Approximately $5 \%$ of HUS cases are associated and triggered by Streptococcus pneumoniae infections (pHUS). ${ }^{3}$ Characteristics of this severe invasive pneumococcal disease (IPD) complication usually become

received

September 13, 2020

accepted after revision

October 20, 2020
DOI https://doi.org/

10.1055/s-0040-1721450. ISSN 2474-5871. apparent 3 to 13 days after the onset of pneumococcal-related symptoms. ${ }^{4}$ Acute mortality is the highest in patients with S. pneumoniae meningitis complicated by pHUS. ${ }^{5}$

Here, we presented a case of a child with pHUS who was successfully treated with tandem hemodiafiltration and plasma exchange. Written informed consent for the publication of the data was obtained from the patient's family.

\section{Case Report}

A previously healthy 22-month-old girl patient with no significant personal medical history was admitted to University Hospital of Split after having fever up to $40^{\circ} \mathrm{C}$ during the past

Copyright $\odot 2020$ Georg Thieme Verlag License terms KG Rüdigerstraße 14, 70469 Stuttgart, Germany
()(1) 
3 days. She previously received all compulsory vaccines. She was diagnosed with left-sided pneumonia. The initial full blood count showed white cell count of $6.1 \times 10^{9} / \mathrm{L}$ (normal $=6$ $16 \times 10^{9} / \mathrm{L}$ ), hemoglobin of $116 \mathrm{~g} / \mathrm{L}$ (normal $=109-138 \mathrm{~g} / \mathrm{L}$ ), and platelet count of $205 \times 10^{9} / \mathrm{L}$ (normal $\left.=150-450 \times 10^{9} / \mathrm{L}\right)$. She had C-reactive protein of $216 \mathrm{mg} / \mathrm{L}$ (normal $=0.1-2.8 \mathrm{mg} / \mathrm{L}$ ), with normal serum creatinine and urea. Urine output was normal. Sepsis was suspected, and crystalloids and ceftriaxone i.v. were started. Her condition initially improved. Since her temperature remained up to $38.8^{\circ} \mathrm{C}$, and due to increase of inflammatory biomarkers (C-reactive protein $226 \mathrm{mg} / \mathrm{L}$, procalcitonin $5.1 \mathrm{ng} / \mathrm{mL}$ [normal $<0.05 \mathrm{ng} / \mathrm{mL}$ ]), vancomycin i.v. was added. Two days later, $S$. pneumoniae susceptible to ceftriaxone and meropenem was confirmed in blood culture. Serotype was not determined. That day, the girl's overall condition deteriorated as she became dyspnoic and anuric, so she was transferred to the pediatric intensive care unit (PICU). Lung ultrasound revealed left-sided pleural effusion of $1 \mathrm{~cm}$. Supplemental oxygen via high-flow nasal cannula was initiated, and ceftriaxone was replaced with meropenem. The full blood count showed hemoglobin of $80 \mathrm{~g} / \mathrm{L}$, and platelet count of $13 \times 10^{9} / \mathrm{L}$. Peripheral blood smear revealed schistocytes. Her serum creatinine was $199 \mu \mathrm{mol} / \mathrm{L}($ normal $=15-31 \mu \mathrm{mol} / \mathrm{L})$, urea was $23.8 \mathrm{mmol} /$ $\mathrm{L}$ (normal $=1-7.5 \mathrm{mmol} / \mathrm{L}$ ), lactate dehydrogenase (LDH) level was 4,046 U/L (normal $=150-360 \mathrm{U} / \mathrm{L}$ ), and she had albumin of $23 \mathrm{~g} / \mathrm{L}$ (normal $=28-48 \mathrm{~g} / \mathrm{L}$ ) and hyponatremia $(128 \mathrm{mmol} / \mathrm{L}$ [normal =134-143 mmol/L]). Other electrolytes were within the normal range, while liver enzymes levels were slightly elevated. Direct antiglobulin (Coombs) test was positive. The $\mathrm{C} 3$ and $\mathrm{C} 4$ were low $(0.5 \mathrm{~g} / \mathrm{L}[$ normal $=0.9-1.8 \mathrm{~g} / \mathrm{L}]$ and $0.05 \mathrm{~g} / \mathrm{L}$ [normal $=0.1-0.4 \mathrm{~g} / \mathrm{L}]$, respectively). C-reactive protein decreased to $189 \mathrm{mg} / \mathrm{L}$, but procalcitonin increased to $11 \mathrm{ng} / \mathrm{mL}$. S. pneumoniae-associated HUC was diagnosed. During perioperative transfusion of platelets, which were administered due to increased risk of bleeding, a dialysis catheter was inserted in right internal jugular vein After that, daily sequence tandem continuous venous hemodiafiltration and therapeutic plasma exchange with albumins (TPE) were started. Transfusion of washed red blood cells (RBC) was administered at erythrocytes level of $2.3 \times 10^{12} / \mathrm{L}$ and hemoglobin level of $56 \mathrm{~g} / \mathrm{L}$. A day after, enlarged left-sided septated pleural effusion was observed. A drain was inserted into pleural cavity and the respiratory status improved after approximately $100 \mathrm{~mL}$ of empyema was evacuated. Over the next 4 days, we were following the improvement of the patient's clinical condition and the laboratory parameters. She received two additional transfusions of unwashed RBCs at hemoglobin levels $<70 \mathrm{~g} / \mathrm{L}$. Platelets continued to fall to $7 \times 10^{9} /$ $\mathrm{L}$, but on the 5 th day increased to $25 \times 10^{9} / \mathrm{L}$, as well as $\mathrm{C} 3$ and C4. Urea was $13.8 \mathrm{mmol} / \mathrm{L}$, creatinine $119 \mu \mathrm{mol} / \mathrm{L}$, and LDH 697 U/L. Urine output gradually improved. TPE was stopped while continuous venovenous hemodiafiltration was continued for five additional days ( - Fig. 1). After 11 days in the PICU, in good condition, she was transferred back to the ward requiring further follow-up. Computed tomographic scan of the thorax revealed necrosis in the upper and lower part of the left lung. She underwent conservative treatment and was discharged from the hospital after a total of 37 days in good condition, with normal blood pressure and with laboratory parameters within

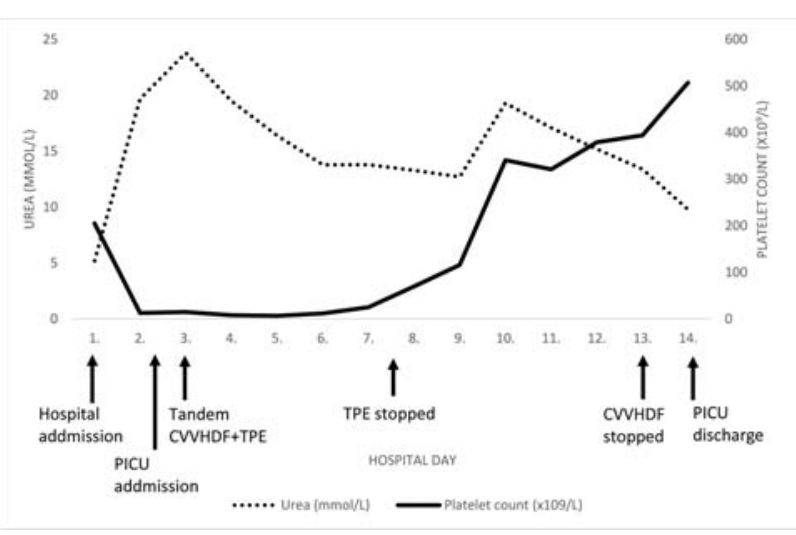

Fig. 1 Urea and platelets changes over the course of illness with the timing of the therapeutic procedures.

normal ranges (urea $5.4 \mathrm{mmol} / \mathrm{L}$ and creatinine $30 \mu \mathrm{mol} / \mathrm{L}$ ). Urinalysis was also normal, without hematuria and proteinuria.

\section{Discussion}

We present the first pediatric case of pHUS treated with sequence tandem continuous venovenous hemodiafiltration and therapeutic plasma exchange in Croatia.

Compared with STEC-HUS, children affected by pHUS are younger with more severe renal and/or hematological disease, and require longer hospital stays. ${ }^{6,7}$ The incidence of pHUS appears to be increasing, ${ }^{8}$ which may be due to increased recognition or possibly a shift in serotype of IPD associated with increased vaccination. ${ }^{4,6}$ Despite being covered by PCV13 vaccine, pneumococcal serotype 19A may still remain the most commonly reported serotype associated with pHUS. ${ }^{8}$ In Croatia, 7-valent pneumococcal conjugate vaccine (PCV) was first introduced in 2010 as a self-sponsored vaccine. Gradually, other conjugate vaccines, including 13-valent, have become available. PCV has been included in the national immunization program since 2019. However, the vast majority of older children remained unvaccinated.

Pathogenesis of pHUS is partially understood and it is not possible to accurately predict which patients with IPD will develop it. ${ }^{6}$ All serotypes of S. pneumoniae have neuraminidase activity capable of exposing the Thomsen-Friedenreich (TF) antigen (T-antigen; TA; Galb1-3GalNAc). Klein et al postulated that the interaction of preformed anti-TF antibodies with the exposed antigen induces hemolysis, platelet agglutination, microvascular thrombosis, and tissue injury. ${ }^{9}$ The proposed mechanism appeared plausible in view of a positive Coombs test seen in approximately 60 to $90 \%$ of pHUS patients. ${ }^{4}$ However, TA exposure alone does not seem to be sufficient in causing pHUS. Other hypothesized mechanisms include decreased sialylation, high concentration of galectin-3, transient dysregulation of complement activation, gene mutations, and disruption of the endothelial cell layer and creation of a local thrombogenic state. ${ }^{6}$ Therefore, it remains unclear whether microbial factors, host factors, or a combination of both contributes to the development of pHUS. ${ }^{6}$

Since the pathogenesis of pHUS is only partially understood, the treatment remains controversial. ${ }^{4,10}$ Usually it starts with 
antibiotic treatment of the pneumococcal infection and other supportive measures. Renal replacement therapy is in patients with pHUS more often required than in those with STEC-HUS (75 vs. 59\%). ${ }^{4}$ Eculizumab has been used, but there is insufficient evidence to recommend it, except in severe cases. ${ }^{6}$

Given that hypothesized involvement of anti-TA antibodies, use of plasmapheresis specifically with albumin to remove the antibodies has been attempted in pHUS patients. It was used mainly for neurologic involvement of the disease, but there is currently no strong systematic evidence that it may improve outcomes to support its efficacy. ${ }^{4,11,12}$

In our patient, 5-day course of plasmapheresis with albumin resulted with platelet count increase and significant improvement of the patient's condition. Due to concern that anti-TA antibodies may be present in blood products, which might worsen hemolysis, plasma, and unwashed RBCs or platelets are avoided in pHUS, as long as agglutination tests are positive. ${ }^{4,11}$ Although the first administered transfusion in our patient was with washed RBCs, the following transfusions were not. Complications or clinical worsening were not noticed; however, Szilágyi et al published a prospective series of five previously healthy children with pHUS. ${ }^{13}$ All were Coombs positive and demonstrated mild-to-moderate depression of serum C3 and C4. Gilbert et al reported two patients with pHUS both demonstrating transiently depressed serum C3, and normal C4 concentrations. ${ }^{14}$ In our patient, improvement was seen regarding depletion of total hemolytic complement activity associated with reduced $\mathrm{C} 3$ and $\mathrm{C} 4$, as well.

Although we implemented multiple interventions, in our opinion, plasmapheresis had beneficial effects and should be considered as a part of standard treatment of children with pHUS. However, a further research is warranted, including a formation of a patient registry to additionally clarify the impact of plasmapheresis. Finally, due to increasing incidence of pHUS and significant burden of IPD, pneumococcal vaccination should be encouraged as well.

\section{Conflict of Interest}

None declared.

\section{References}

1 Fakhouri F, Zuber J, Frémeaux-Bacchi V, Loirat C. Haemolytic uraemic syndrome. Lancet 2017;390(10095):681-696

2 Raina R, Krishnappa V, Blaha T, et al. Atypical hemolytic-uremic syndrome: an update on pathophysiology, diagnosis, and treatment. Ther Apher Dial 2019;23(01):4-21

3 Cody EM, Dixon BP. Hemolytic uremic syndrome. Pediatr Clin North Am 2019;66(01):235-246

4 Spinale JM, Ruebner RL, Kaplan BS, Copelovitch L. Update on Streptococcus pneumoniae associated hemolytic uremic syndrome. Curr Opin Pediatr 2013;25(02):203-208

5 Banerjee R, Hersh AL, Newland J, et al; Emerging Infections Network Hemolytic-Uremic Syndrome Study Group. Streptococcus pneumoniae-associated hemolytic uremic syndrome among children in North America. Pediatr Infect Dis J 2011;30(09): 736-739

6 Scobell RR, Kaplan BS, Copelovitch L. New insights into the pathogenesis of Streptococcus pneumoniae-associated hemolytic uremic syndrome. Pediatr Nephrol 2019. Doi: 10.1007/s00467019-04342-3

7 Salvadori M, Bertoni E. Update on hemolytic uremic syndrome: diagnostic and therapeutic recommendations. World J Nephrol 2013;2(03):56-76

8 Lawrence J, Gwee A, Quinlan C. Pneumococcal haemolytic uraemic syndrome in the postvaccine era. Arch Dis Child 2018;103 (10):957-961

9 Klein PJ, Bulla M, Newman RA, et al. Thomsen-Friedenreich antigen in haemolytic-uraemic syndrome. Lancet 1977;2(8046):1024-1025

10 Loirat C, Saland J, Bitzan M. Management of hemolytic uremic syndrome. Presse Med 2012;41(3 Pt 2):e115-e135

11 Copelovitch L, Kaplan BS. Streptococcus pneumoniae-associated hemolytic uremic syndrome. Pediatr Nephrol 2008;23(11): 1951-1956

12 Schwartz J, Padmanabhan A, Aqui N, et al. Guidelines on the use of therapeutic apheresis in clinical practice-evidence-based approach from the writing committee of the American Society for Apheresis: the seventh special issue. J Clin Apher 2016;31(03): 149-162

13 Szilágyi A, Kiss N, Bereczki C, et al. The role of complement in Streptococcus pneumoniae-associated haemolytic uraemic syndrome. Nephrol Dial Transplant 2013;28(09):2237-2245

14 Gilbert RD, Nagra A, Haq MR. Does dysregulated complement activation contribute to haemolytic uraemic syndrome secondary to Streptococcus pneumoniae? Med Hypotheses 2013;81(03): 400-403 